\title{
粗粒内源性网络可预测血液系统髓系细胞分化和 转分化的主要时序特征
}

\author{
苏杭 ${ }^{1}$, 王高伟 ${ }^{1}$, 袁若石 ${ }^{1,2}$, 敖平 $1,2,3,4 *$ \\ 1. 上海交通大学系统生物医学研究院, 系统生物医学教育部重点实验室, 上海 200240; \\ 2. 上海交通大学生物医学工程学院, 上海 200240 ; \\ 3. 上海交通大学医学院, 上海肿瘤研究中心国家重点实验室, 上海 200240 ; \\ 4. 上海大学定量生命科学国际研究中心和物理系, 上海 200444 \\ * 联系人, E-mail: aoping@sjtu.edu.cn
}

收稿日期: 2017-02-20; 接受日期: 2017-05-11; 网络版发表日期: 2017-06-13

国家自然科学基金(批准号: 91529306, 91329301, 91652930016)资助

\begin{abstract}
摘要揭示细胞命运决定过程中的分子调控机制对理解干细胞分化和组织稳态的建立和维持具有决定性的 意义. 本文通过归纳从机制研究中积累的分子调控关系, 构建了血液系统髓系细胞分化相关的粗粒化内源性网 络. 通过对网络动力学进行定量分析, 本文以稳态的概念对髓系主要细胞类型进行分类, 并定量预测了一系列 细胞类型转换过程中出现的中间状态或过渡态. 基于计算结果和数学中动力学系统的原理, 本文提出了髓系细 胞发育景观拓扑图, 并从高维非线性动力系统的基本特性出发, 讨论了实验中在特定基因操作下观测到的转 分化现象, 并预测了相应过程中的分子动力学变化. 本研究表明, 髓系细胞发育定量景观拓扑图的构建有助于 全局性地阐述恶性肿瘤和再生障碍性疾病的发生发展。
\end{abstract}

关键词造血系统, 发育景观, 内源性网络, 拓扑结构, 稳态, 过渡态, 动力学建模

血液是人体中一类快速进行自我更新的组织, 包 含诸多具有不同功能的细胞类型. 由于血细胞本身流 动的生活模式, 及其易分离易培养等诸多优良特性, 被广泛应用于研究干细胞分化和细胞命运决定过程 中, 并积累了大量研究成果. 在过去的研究中, 根据细 胞表面抗原的表达, 人们从实验中分离出造血干细胞 (hematopoietic stem cells, HSCs)和祖细胞(progenitors), 并根据其功能分析结果构建了经典的造血发育谱系 图 ${ }^{[1]}$. 血液细胞来源于共同的祖先, 即长期造血干细胞 (long-term hematopoietic stem cells, LT-HSCs). LT-HSCs
具有持续的自我更新能力, 以保证血液系统功能细胞的 持续性补充. 在其分裂过程中, 一部分子细胞会继续保 持干性, 余下的细胞则会根据细胞内外的环境和情况, 选择不同的细胞命运: LT-HSCs会分化产生短期造血 干细胞(short-term hematopoietic cells, ST-HSCs)或多能 祖细胞(multi-potent progenitors, MPPs), 这些细胞状态 的复制能力进一步降低, 并产生不同的发育潜能; MPPs 会进一步分化, 产生一些谱系特异性的前体细胞, 如定 向髓系祖细胞(common myeloid progenitors, CMPs)或 定向淋系祖细胞(common lymphoid progenitors, CLPs);

引用格式: 苏杭, 王高伟, 袁若石, 等. 粗粒内源性网络可预测血液系统髓系细胞分化和转分化的主要时序特征. 中国科学: 生命科学, 2017, 47: 616-628 Su H, Wang G W, Yuan R S, et al. Coarse-grained endogenous network predicts main dynamical sequences in myeloid differentiation and trans-differentiation. Sci Sin Vitae, 2017, 47: 616-628, doi: 10.1360/N052016-00309 
CLPs不具备分化为髓系细胞的潜能, 但能够分化产生 所有的淋系细胞; CMPs会进一步分化为巨核-红系祖 细胞 (megakaryocyte-erythrocyte progenitors, MEPs) 或 粒系-单核系祖细胞 (granulocyte-monocyte progenitors, GMPs). 这些双能祖细胞根据细胞内外特定的诱导或 噪声影响最终分化为红细胞、能够产生血小板的巨 核细胞或粒细胞、单核-巨噬细胞. 这一经典的造血 发育谱系图强调了组织发育过程中的一些基本特点, 如单向性、层次性和步进式命运决定等, 并成为理解 干细胞分化的范例 ${ }^{[2]}$. 同时, 它也是一个标准生物模型 来揭示细胞命运决定过程中的分子调控机制, 研究它 对于理解正常组织的发育和复杂疾病的发生发展具 有极其重要的意义.

在这样高度层次分明的发育过程背后, 调控细胞 命运的分子机制是什么? 在过去的 20 年中, 大量的工 作试图揭示造血发育过程中决定细胞命运的分子调 控机制. 一系列实验上的发现显示, 造血发育的调控 是由大量遗传和表观遗传调控因子的相互作用所决 定的 ${ }^{[3]}$. 成百上千的调控因子之间通过相互作用形成 一个复杂调控网络, 接收处理内源、外源信号并进行 细胞命运的选择. 然而, 生物系统的复杂性使得一些 特征无法仅从单个调控因子的研究中推断出来. 理解 单个因子活性的变化如何影响整个调控网络, 从而对 表型产生影响的动力学过程依然是一个重要挑战.

关于细胞状态和细胞分化路径, 历史上曾提出很 多相关的概念模型, 如 20 世纪, Waddington ${ }^{[4]}$ 提出了发 育景观学说, 将细胞发育和干细胞分化过程比喻成管 道系统: 细胞在不同的分叉口选择不同的路径, 从而 落入不同细胞命运. 基于细胞调控网络的动力学分析, 人们对发育过程从定量的角度提出新的景观学理解, 并以稳态的概念来解读不同细胞状态的维持 ${ }^{[5]}$. 之后, 内源性网络理论提出了一套可行的方法来对生物系统 中核心分子调控网络进行概括性描述和定量分析 ${ }^{[6-8]}$, 并进一步应用于包括癌症在内的许多复杂疾病的研 究中 ${ }^{[6,7,9 \sim 12]}$, 从随机动力学的角度重新解读其发生发 展过程 ${ }^{[13,14]}$, 并得出一系列可被实验验证的预测. 在内 源性网络的理论框架下, 本文通过总结在长期的研究 中所积累的分子调控机制, 构建与髓系细胞分化相关 的粗粒化内源性网络; 通过将网络进行定量描述和动 力学分析, 得到了一组由网络结构所决定的态(state); 根据每个态对局域扰动敏感性的不同, 这些态被分为
稳态和不稳定 (或过渡态); 将模型与独立的实验数据 进行对比, 结果表明内源性网络产生的稳态分别对应 不同的细胞类型, 而过渡状态对应着一些发育过程中 经历的中间状态; 通过对网络动力学进行进一步分析, 讨论了态与态之间转换的可能路径, 并模拟了在不同 的基因操作下细胞转分化的过程. 内源性网络动力学 预测了在特定基因操作下细胞表型的变化,一部分预 测得到了实验中在相应的基因操作下观测到的转分 化现象的支持. 模型进一步预测了在特定的细胞类型 转换过程中, 不同调控因子的含量或活性随时间的动 力学变化, 从而为调控造血发育过程中细胞分化和转 分化提供新的思路.

部分分子生物学家很早就意识到要从网络角度 来考察复杂生物过程, 并要考虑其主要因素. 其中一 个代表性的论证是所谓癌症主要标志假说 ${ }^{[15]}$. 该假说 直接有粗粒化的层次. 有意思的是主要标志假说目前 得到很大赞同, 网络假说却没有得到充分认可. 在前 期工作中, 本课题组对这个问题进行了深入研究, 提 出的内源性网络理论不但发展这个思路, 并进一步表 明(非正常的)免疫因素, 包括炎症状反应和(非正常的) 代谢因素都应该考虑进来 ${ }^{[6]}$. 尽管基因组不稳定和变 异、以及染色体数目异常和癌症相关性很强, 分析表 明它们不是癌症的主要机制, 并可能从内源性网络理 论得到解释 ${ }^{[16]}$. 这 4 个相应的标志物构成了几年以后 癌症主要标志第二代假说的主要内容 ${ }^{[17]}$. 在过去 10 年 中内源性网络理论也得到很大发展, 现已能与癌症的 临床数据直接比对 ${ }^{[9 \sim 12]}$. 作为起点, 本文主要讨论了内 源性网络的最核心的一部分, 下面称之为“粗粒内源 性网络”, 以求能对主要现象做出定量、时序的描叙 和预测.

\section{1 材料与方法}

\section{1 常微分方程动力系统, 平衡点计算算法:}

常微分方程组 (ordinary differential equations, ODEs) 由内源性网络结构转化而来, 描述了每个因子 在其他因子的调控下随时间进行动力学变化的过程. 本文采用了两种独立的算法来计算常微分动力系统 中的平衡点. 以如下动力系统为例:

$$
\frac{\mathrm{d} x(t)}{\mathrm{d} t}=f(x)
$$


(1) 算法一. 这一算法可以用于计算稳定平衡点, 通过随机产生一个八维的向量 $x_{0}$ 作为系统的初始值, 使向量 $x_{0}$ 随时间在 $x_{t+1}=x_{t}+\Delta \times f\left(x_{t}\right)$ 条件下迭代. 在足够 长时间的迭代后, 以 $\left(x_{t+1}-x_{t}\right)^{2}<\varepsilon, f\left(x_{t}\right)^{2}<\delta$ 来判断系统是 否收玫, 参数取值如下 $\Delta t=0.1, N=800, \varepsilon=10^{-8}, \delta=10^{-8}$. 如 果迭代后的向量 $x_{t}$ 满足上述条件, 则将向量 $x_{t}$ 记为系 统产生的一个稳态. 如选取 1000 次随机系统初始值, 记录所有计算得到的稳态; 如果重复取样的次数在 10000000 或更多的情况下, 计算依然得到同样的计算 结果, 则将以上计算得到的稳态作为模型初步的计算 结果.

(2) 算法2. 该算法利用牛顿迭代法求解非线性方 程 $\frac{\mathrm{d} x(t)}{\mathrm{d} t}=f(x)=0$ 来计算稳定平衡点和不稳定平衡点. 通过随机产生一个八维的向量 $x_{0}$ 作为系统的初始值, 利 用牛顿迭代法对初始向量进行迭代, 找到满足 $f\left(x^{*}\right)=0$ 的 解 $x^{*}$. 具体而言, 本文利用矩阵实验室Matlab中的函数 fsolve对动力系统进行求解. 如果方程 $f\left(x^{*}\right)=0$ 在 $x^{*}$ 处 的雅克比矩阵的所有特征值均为负, 则 $x_{i}$ 被判定为稳 态; 如果方程 $f\left(x^{*}\right)=0$ 在 $x^{*}$ 处的雅克比矩阵的所有特 征值至少有一个为正, 则 $x_{i}$ 被判定为不稳定状态. 每 一个不稳定状态所对应的雅克比矩阵中正的特征值 的个数也被记录下来. 通过重复对系统初始值随机取 样, 如 10000 次, 记录所有计算得到的稳态与不稳定状 态; 如果随机取样的次数重复更多次, 如 100000 或更 多, 计算依然得到同样的结果, 则判定稳态与不稳态 个数已经基本稳定. 模型结果显示动力系统参数在合 理的范围内取值, 得到的结果非常稳健.

\section{2 模型计算结果与表达数据对比方法}

为了对模型结果在分子层次上进行进一步的验 证, 将稳态N1, N2, N3, N4, N5 的因子表达量与取自人 体血液样本的功能细胞造血干细胞、粒细胞、红细 胞和巨核细胞的RNA-seq表达数据进行对比. 根据细 胞表面蛋白的特异性表达, 在人体血液样本中分离出 38种不同的细胞类型, 代表了造血干细胞, 祖细胞和末 端分化的功能性细胞类型. 这38中细胞类型通过 211 Affymetrix array U133A测量和分析了其基因表达, 每 一种细胞有4 7个平行. 针对样本的全局表达谱, 每一 个基因的表达量作归一化处理, 使之均值为 0 . 本文设 定 0 为阈值判断每一个基因的高表达或者低表达情况, 从而与网络计算结果进行比较. 每一种细胞类型选取
了 4 个平行样本分别对理论计算值进行验证. RNA-seq 表达数据来源于 $h t t p: / / n c b i . n l m . n i h . g o v / g e o / G S E 24759$.

\section{3 不稳定平衡点局域扰动}

在ODE动力系统中, 当网络处于某一不稳定平衡 点时, 以一个小的随机向量 $\Delta x(|\Delta x|<0.005)$ 对系统进行 局部微扰, 并以扰动后的状态作为初始值, 使之在动力 方程组 $x_{t+1}=x_{t}+\Delta x \times f\left(x_{t}\right)$ 的约束下随时间进行迭代. 结果 记录了扰动后的系统随时间演化的路径, 并记录了最终 系统收玫到的稳态, 判断收玫的条件如下: $\left(x_{t+1}-x_{t}\right)^{2}<\varepsilon$, $f\left(x_{t}\right)^{2}<\delta$. 这里选用的参数值为 $\Delta t=0.1, N=800, \varepsilon=10^{8}$, $\delta=10^{8}$. 随机扰动实验重复多次后, 如 1000 次, 得到某 一特定不稳定平衡点扰动后能够演化到的稳态, 当随 机扰动重复更多次后, 如果依然得到了同样的结果, 则判定结果已经收敛.

\section{2 结果}

\section{1 髓系细胞分化相关的粗粒内源性网络}

在造血发育过程中, 细胞分化和命运决定受到严 格调控以维持血液组织稳态平衡. 虽然造血发育过程 中涉及诸多遗传和表观遗传调控因子的作用, 但在过 去的研究中发现, 少数谱系特异性转录因子的表达与 否决定了发育过程中细胞分化的方向, 是决定细胞命 运的关键调控因子 (master regulator) ${ }^{[18-20]}$. 例如, 在髓 系细胞分化早期, 初级决策者PU.1和GATA1之间的竞 争性表达决定了细胞粒系和红系之间的命运抉择: 当 PU.1高表达时, CMPs向粒系-单核巨嘜细胞系分化; 当 GATA1高表达时, CMPs向巨核-红系细胞系分化. 在这 两个双能祖细胞各自的命运分叉点上, 次级决策因子 的竞争性表达决定了进一步分化方向: 在GMPs时期, Gfi-1和Egr/Nab之间的相互竞争决定了细胞最终分化 形成粒细胞或单核/巨噬细胞; 在MEPs时期, EKLF与 Fli-1之间的相互竞争决定了 MEPs最终分化为红细胞 或者巨核细胞. 此外, 实验中观测到的不同细胞系之 间重分化和转分化现象进一步证明这些谱系特异性 的转录因子在细胞命运决定中的重要作用. 例如, 通 过将 $\mathrm{MyoD}$ 蛋白在成纤维细胞中表达, 可以使成纤维 细胞转换为肌肉细胞 ${ }^{[21]}$; 最近实验中通过将 4 个关键 的转录因子在体细胞中表达所产生的iPS细胞更进一 步证明了这一观点 ${ }^{[22]}$. 因此, 谱系特异性的转录因子 
的表达集合可以视为不同细胞类型在分子层次上的 定义 ${ }^{[20,23]}$.

内源性网络被定义为生物系统在长期演化过程中 形成的保守而自治的核心网络, 实现并调控与之相关 的生物功能. 本文通过归纳和总结造血分化研究中积 累的分子调控机制, 选择了与髓系细胞命运决定相关 的一组关键调控因子对相关内源性网络进行概括性 描述(图1), 包括早期造血干细胞相关的因子(GATA-2, C/EBPs)、初级命运决策因子(GATA-1, PU.1), 以及次 级命运决策因子(EKLF, Fli-1, Egr/Nab, Gfi-1). 这些 因子之间通过直接或间接的激活抑制关系相互调节 (表1和2), 形成了一组保守的、封闭的、执行具体功 能的内源性网络(图2). 因子和因子之间相互作用关系 是在长期的机制研究中总结的结果, 被多

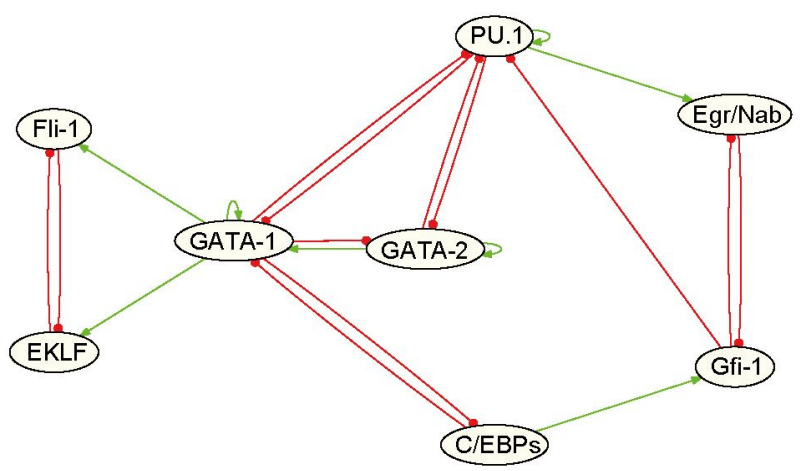

图 1 髓系细胞分化相关的粗粒内源性网络 (网络版彩图) 根据积累的生物学知识, 本文选择了一组髓系细胞分化相关的谱 系特异性转录因子作为内源性网络 ${ }^{[6]}$ 的节点. 因子与因子之间的 相互作用是从已有的基因调控知识中总结而来的 ${ }^{[19]}$. 箭头表示激 活或上调关系, 圆点表示抑制或者下调关系

表 1 髓系细胞分化相关的粗粒内源性网络节点

\begin{tabular}{cc}
\hline Key agent & Description \\
\hline GATA-2 & Transcription factor GATA-2 \\
PU.1 & Transcription factor PU.1, SP-1 \\
Egr/Nab & Transcription factor Egr-1, Egr-2, Nab-2 \\
Transcription factor CCAAT \\
C/EBPs & Enhancer-binding Protein Family \\
& Transcription factor Growth factor \\
Gfi-1 & independence 1 \\
& Erythroid Kruppel-like factor \\
EKLF & (ELKF/KLF1) \\
GATA-1 & Transcription factor GATA-1, FOG-1 \\
Fli-1 & Transcription factor FLI-1: a member \\
& of ETS family \\
\hline
\end{tabular}

表 2 粗粒内源性网络中因子与因子之间的相互作用

\begin{tabular}{|c|c|c|}
\hline Key agents & Activated by & Inhibited by \\
\hline GATA-2 & GATA- $2^{[24]}$ & $\begin{array}{c}\text { PU. } 1^{[25]} \\
\text { GATA-1 }{ }^{[26]}\end{array}$ \\
\hline PU.1 & PU. $1^{[27]}$ & $\begin{array}{c}\text { GATA-1 }^{[28]} \\
\text { GATA-2 }{ }^{[25]} \\
\text { Gfi- }^{[29]}\end{array}$ \\
\hline Egr/Nab & PU. $1^{[30]}$ & Gfi- $1^{[30]}$ \\
\hline C/EBPs & & GATA-1 $^{[31]}$ \\
\hline Gfi-1 & $\mathrm{C} / \mathrm{EBPs}^{[29]}$ & $\mathrm{Egr} / \mathrm{Nab}^{[30]}$ \\
\hline EKLF & GATA- $1^{[32]}$ & Fli $^{[33]}$ \\
\hline GATA-1 & $\begin{array}{l}\text { GATA-2 }{ }^{[32]} \\
\text { GATA- }{ }^{[34]}\end{array}$ & $\begin{array}{l}\text { PU.1 }{ }^{[28,35]} \\
\text { C/EBPs }\end{array}$ \\
\hline Fli-1 & GATA-1 ${ }^{[36]}$ & $\operatorname{EKLF}^{[33]}$ \\
\hline
\end{tabular}

种实验手段和现象所验证, 如基因敲除、过表达、表 达分析等 ${ }^{[19]}$. 相关的基因敲除模拟揭示了简化模型中 因子选择的必要性(网络版附图1).

\section{2 定量描述和强健性分析}

(1) 网络的布尔动力学实现. 对于图1中的粗粒内 源性网络, 由于网络结构的复杂性和节点-节点之间非 线性的相互作用,一些网络动力学特性很难从直观或 线性的逻辑推理中获得. 数学模型的建立提供了一种 有效的办法来对上述非线性复杂网络进行定量分析.

本文首先运用离散动力系统——布尔网络分析 法来对内源性网络进行分析. 布尔网络分析是一种常 见的非线性动力系统分析方法,被广泛运用于复杂生 物系统的描述和分析中 ${ }^{[37,38]}$. 在布尔网络中, 每个节点 的表达只有 0 和 1 两种形式, 分别对应节点的不表达和 高表达. 节点表达量所组成的 8 维向量描述了系统在 $t$ 时刻的状态. 在布尔网络分析中, 系统状态随离散的 时间点进行迭代, 在 $t+1$ 时刻每个因子的含量是由 $t$ 时 刻的系统状态所决定的 $x_{t+1} \leftarrow f\left(x_{t}\right)$. 状态迭代所遵循的 布尔法则是由网络结构转化而来: 网络中节点之间的 激活抑制关系可以用and, or, not 3 种布尔逻辑式表示 出来, 如在图1中, GATA-2受自身的激活, 及GATA-1和 PU.1的抑制, 三者关系可转化为以下逻辑关系式:

$x_{\text {Gata-2 }}(t+1)=x_{\text {Gata-2 }}(t) \operatorname{and}\left(n o t x_{\text {Gata-1 }}(t)\right) \operatorname{and}\left(n o t x_{\mathrm{PU}, 1}(t)\right)$.

类似的, 其他 7 个节点之间的相互作用也可以通过 

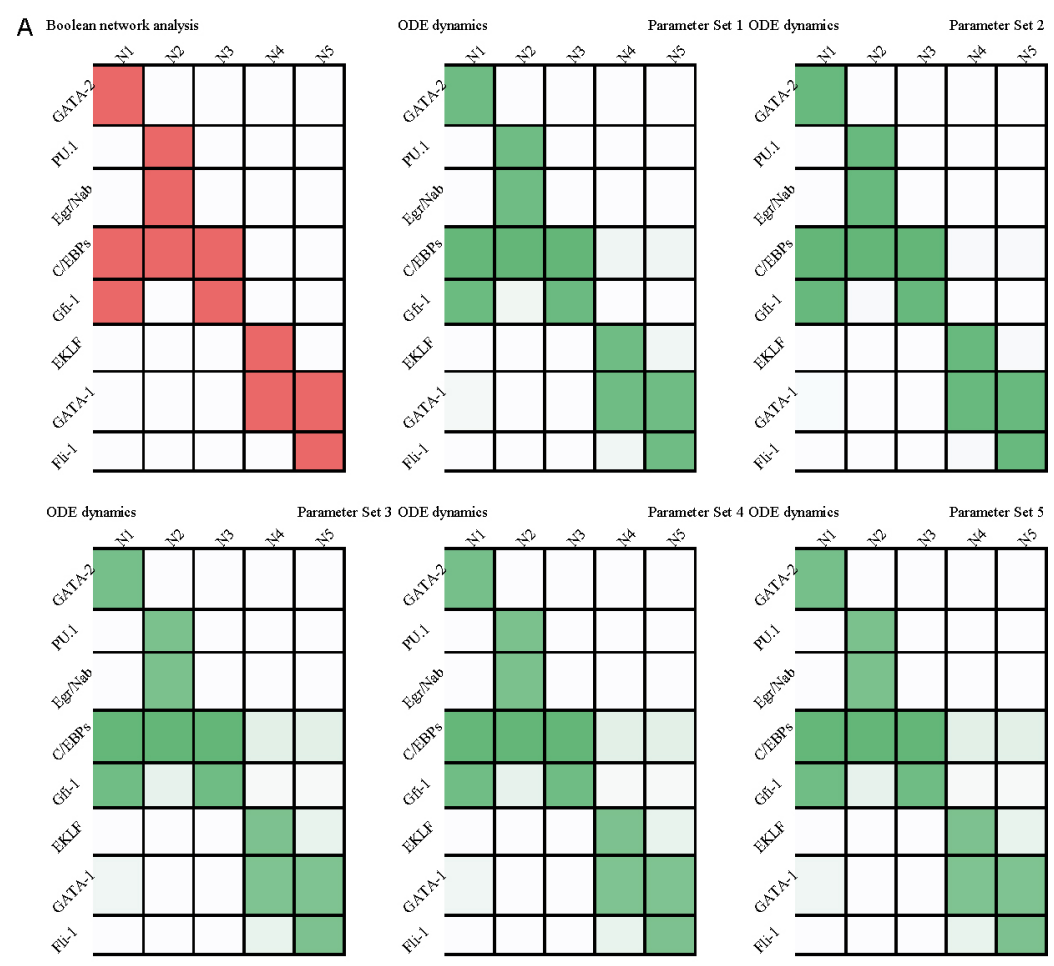

B
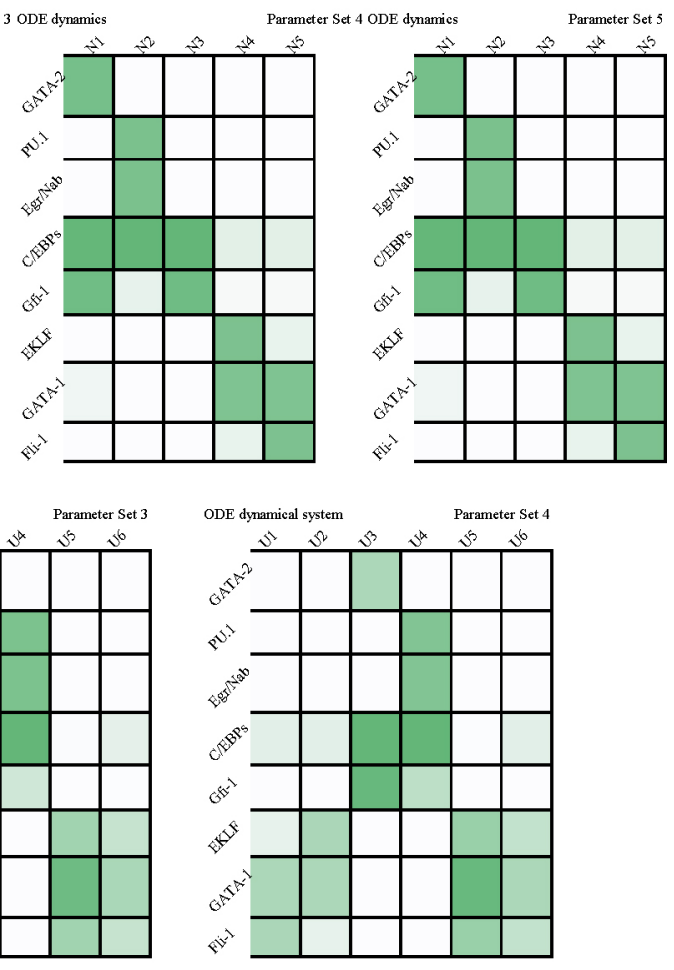

ODE dynamical system

(1)
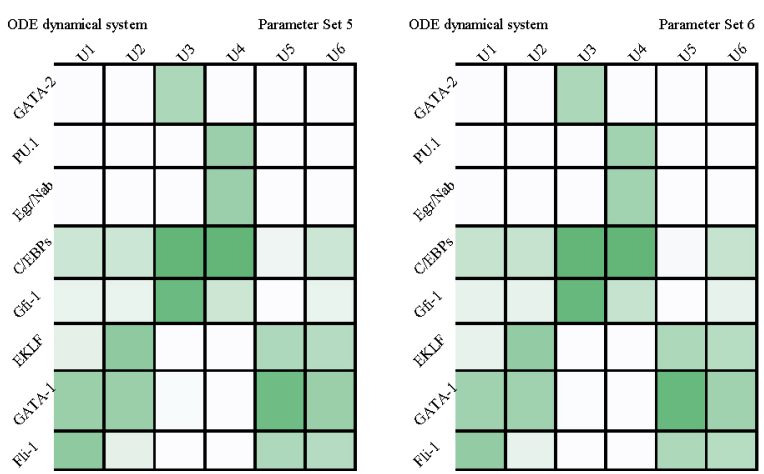

图 2 内源性网络中状态的强健性(网络版彩图)

A: 内源性网络结构产生的稳态. 通过布尔网络分析和不同参数条件下ODE动力系统分析, 发现如图 5 个稳态始终非常稳定地存在. 在布尔 网络分析结果中, 深色代表因子表达为 1 , 浅色代表因子表达为 0 ; 在ODE动力学分析结果中, 深色代表因子表达为 1 , 浅色代表因子表 达为 0 ; B: 内源性网络结构产生的不稳定状态. 在不同参数条件下进行ODE动力系统分析, 发现 6 个不稳定状态始终存在. 深色代表 
布尔逻辑关系表示出来(网络版补充材料). 通过随机 地选取一些8维的初始值, 使不同的初始状态在这些 逻辑关系的约束下随时间不断演化, 可以发现系统会 逐步收玫到一些特定的稳态中. 如果通过对初始值进 行 1000 次随机取样后, 在动力系统中共找到了 5 个稳 态, 当随机次数达到 10000 次或更多的情况下, 依然只 找到了 5 个相同的稳态, 则判定稳态数量已经收玫. 每 个稳态对应着一组 8 维的向量(图2), 表示在不同的细 胞状态下 8 个转录因子的不同表达量. 本文进一步发 现, 内源性网络在两种不同的布尔逻辑式的实现形式 下(网络版补充材料), 一部分稳态始终很稳定的存在 (网络版附表1和2). 动力系统的一些其他复杂特性, 如 极限环等, 在这一步的工作中暂时不作讨论.

(2) 网络的常微分方程动力系统实现. 本文进 一步用一组常微分方程(ordinary differential equations, ODEs)来描述每一个因子的含量在其他因子的调控下 随时间的动态变化. 本文采取了无量纲的建模框架, 将每一个因子的表达值归一化到0 1.0代表因子完全 不表达, 1 代表因子最高表达状态. 由于希尔方程形式 被广泛应用于描述生物系统中的基因调控过程 ${ }^{[39]}$, 本 文采用希尔方程作为基本形式来描述因子之间的非 线性相互作用关系. 以因子 $x$ 为例, 描述其含量变化的 常微分方程(2)所示:

$$
\frac{\mathrm{d}[x(t)]}{\mathrm{d} t}=f(X)-\frac{[x(t)]}{\tau_{x}},
$$

其中, $[x(t)]$ 代表 $t$ 时刻的浓度或活性; $\frac{[x(t)]}{\tau}$ 表示在 $t$ 时刻 因子 $x$ 降解或失活的速率; $f(X)$ 代表在自身及其他因子 的调控作用下因子 $x$ 的生成速率, 其中 $X$ 代表了对因子 $x$ 具有调控作用的因子含量或活性所组成的向量. $f(X)$ 可用以下式子进行表示:

$$
f(X)=V_{\max } \times \frac{k_{a} \times \sum x_{a}{ }^{{ }_{a}}}{1+k_{a} \times \sum x_{a}{ }^{{ }^{a}}} \times \frac{1}{1+k_{i} \times \sum x_{i}{ }^{{ }}{ }},
$$

或:

$$
f(X)=V_{\max } \times \frac{k_{a} \times \sum x_{a}{ }^{{ }{ }_{a}}}{1+k_{a} \times\left(\sum x_{a}{ }^{{ }_{a}+} \sum x_{i}{ }^{{ }}\right)},
$$

其中, $V_{\max }$ 代表因子 $x$ 最大合成速率, $x_{a}$ 代表所有对 $x$ 具 有激活作用的因子浓度或活性, $x_{i}$ 代表所有对 $x$ 具有抑 制作用的因子浓度或活性, $k_{a}, k_{i}$ 分别为反映激活和抑
制反应的解离常数, $n_{a}, n_{i}$ 为相关激活或抑制反应的希 尔系数. 在这一无量纲的建模框架下, 最大产生速率 $V_{x}$ 和最大降解系数 $\tau_{x}$ 归一化为 1 . 希尔系数 $n$ 和解离常 数 $k$ 在合理的范围内变化以描述因子与因子之间的激 活抑制关系的基本特征, 并设定了条件 $k \approx 2^{n}$ 来约束 $n$ 与 $k$ 之间的关系, 从而将 $\mathrm{S}$ 型曲线的阈值归一化到 0.5 附 近. 如当 $n$ 取 3 时, $k$ 在 8 附近取值 $(k=8,9,10 \ldots)$. 当希尔 系数 $n$ 趋向于正无穷时 $n \rightarrow+\infty$, 微分方程所描述的因子 与因子之间 $\mathrm{S}$ 型的变化曲线与布尔网络分析方法类似 (本文假设简化网络中因子与因子之间的相互作用是 等权重的, 激活与抑制关系之间抑制关系更强). 内源 性网络在ODE动力系统的表达关系式见附录.

通过以上方法, 将构建的粗粒内源性网络转化为 一组非线性常微分方程, 并对其进行动力学分析(见材 料与方法), 模拟网络随时间演化的过程, 寻找网络的 平衡点. 本文采用两种独立的方程形式对网络结构进 行描述, 并使参数在合理的范围内进行变化, 结果表 明网络在不同的方程形式和参数条件下,一部分态始 终非常稳定的存在(网络版补充材料), 即在两种不同 的方程形式、合理的参数条件和两种独立的算法下 始终能够得到 5 个稳态和 6 个不稳定状态(图2). 对于在 常微分方程动力系统中计算得到的稳态而言, 当设定 阈值将每一个因子的表达量判定为 0 或 1 , 即因子含量 大于阈值时为判定为 1 , 小于阈值时为判定为 0 时, 结 果表明, 常微分方程动力系统计算得到的稳态与布尔 网络分析中得到的稳态完全一致. 阈值的取值可以在 0.3 0.7变化, 而不会影响最终的结果. 此外, 前期的工 作发现在一种新的积分方式下, 通过常微分方程组计 算得到的平衡点与对应的随机微分方程组(stochastic differential equations, SDEs)计算的结果完全一致 ${ }^{[13]}$. 这 些计算结果进一步支持和验证了已往关于生物系统 稳定性的研究 ${ }^{[40,41]}$, 即网络的拓扑结构本身决定了系 统状态的稳定性和适应性, 同时也反映了依照内源性 网络理论所构造网络的合理性及可行性, 从而为进一 步研究奠定基础.

(3) 稳态和过渡态的生物学意义. 生物中的景观 概念自 20 世纪早期提出以来 ${ }^{[4,42]}$, 在复杂系统的定量描 述中得到了广泛的应用和发展 ${ }^{[5]}$, 表型转换过程中遗 传和表观遗传调控因子的作用在此框架下有了新的 理解和讨论 ${ }^{[43-45]}$. 景观学中稳态的概念被广泛应用于 理解细胞的不同状态及状态转移中 ${ }^{[6]}$, 并作为一个桥 
梁, 将分子机制和表型联系在一起. 每一个稳态对应 着网络中特定的一组因子表达值, 在生物中可对应着 在某一细胞状态下不同的转录因子的活性或表达量. 根据网络中因子在生物系统中的特殊功能, 可以通过 分析模型结果中每个稳态下因子的特异性表达, 来推 断其可能对应的生物学意义.

像上文讨论过的, 在干细胞分化过程中, 谱系特 异性转录因子的表达与否对细胞命运的决定起到了 关键的调控作用. 例如, 在髓系细胞分化早期, 初级 决策者PU.1和GATA1之间的竞争性表达决定了细胞 粒系和红系之间的命运抉择: 当PU.1高表达时, CMPs 向粒系-单核巨噬细胞系方向分化; 当GATA1高表达 时, CMPs向巨核-红细胞系分化. 在GMPs时期, PU.1 和 $\mathrm{C} / \mathrm{EBP} \alpha$ 的相对含量高低, 以及 Gfi-1和 Egr/Nab之间 的相互竞争决定了细胞最终分化形成粒细胞或单核 /巨噬细胞 ${ }^{[29,46]}$; 而在MEPs 时期, EKLF与Fli-1之间的 相互竞争决定了MEPs最终分化为红细胞或者巨核细 胞 $^{[29,36]}$. 因而, 特异性转录因子的共表达可以作为细 胞类型在分子层次上的定义, 从而对细胞类型进行划 分. 模型结果表明, 网络计算得到的稳态因子表达很 好地对应了不同细胞类型在分子层次上的定义: 稳态 N2描述了单核-巨噬细胞, 即PU.1(高比例), C/EBPa(低 比例)和 Egr/Nab共表达的状态; 稳态N3描述了粒细胞, 即 C/EBP $\alpha$ (高比例), PU.1(低比例)Gfi-1 的共表达状态; 稳态N4, 描述了红细胞GATA-1和EKLF共表达的状态; 稳态N5描述了巨核细胞GATA-1和Fli-1共表达的状态, 稳态 $\mathrm{N} 1$ 对应着其他粒细胞的分子特征 ${ }^{[4]}$.

进而, 本文将网络计算得到的 5 个稳态的因子 表达量与实验中取自人类血液样本中对应的细胞类 型(单核-巨噬细胞、两种粒细胞、红细胞和巨核细 胞)RNA-seq数据 ${ }^{[48]}$ 进行对比, 以对网络计算结果进行 进一步验证(见材料与方法). 分析结果表明, 网络计 算结果中不同的稳态与相应的细胞类型表达数据的 符合程度分别为 $68.8 \%, 87.5 \%, 75 \%, 84.4 \%, 75 \%$ (图3). 由于稳态中的因子表达是直接通过网络计算得来, 而 高维非线性动力系统中稳态特征是很难进行操控或 拟合的, 这一结果表明, 网络计算得到的不同稳态对 应着血液细胞发育过程中的不同细胞类型.

本文进一步分析了由网络结构本身所决定的不 稳定平衡点的生物学意义, 发现不稳定平衡点可能对 应着细胞类型的中间状态. 从生物学机制上而言, 在
早期发育过程中, 初级命运决策者PU.1 和 GATA-1 竞 争性表达决定了CMPs向 GMPs或MEPs 分化. 当PU.1 高表达的情况下, GMPs的进一步细胞命运决定由次 级命运决策者 Egr/Nab 与 Gfi-1 的竞争性表达执行: 当 Egr/Nab高表达的情况下, 祖细胞向单核一巨噬细胞分 化; 当Gfi-1高表达的情况下, 祖细胞向粒细胞分化. 在 网络计算结果中, 不稳定平衡点部分因子表达处于中 间水平, 即在阈值 $0.3 \sim 0.7$ 范围内. 其中, 两个不稳定 平衡点的因子表达与在发育过程中相应因子相互竞 争的中间状态有很好的一致性: 不稳定状态U4中在 初始命运决定转录因子PU.1和C/EBPs高表达的情况 下, Egr/Nab和Gfi-1的表达值处于阈值(0.3 0.7)范围内, 与粒细胞-单核/巨噬细胞祖细胞时期的细胞命运决 定过程有着很好的对应. 类似的, 当早期命运决策者 GATA-1 高表达的情况下, MEPs进一步的细胞命运决 定由EKLF与Fli-1的竞争性表达决定: 当Fli-1高表达 时, 祖细胞向巨核细胞分化; 当EKLF高表达时, 祖细 胞向红细胞分化. 网络计算结果中不稳定状态U5的因 子表达值则与巨核-红系祖细胞时期细胞命运决策状 况十分一致, 即早期细胞命运决策者GATA-1 高表达, 而EKLF和Fli-1的表达值处于阈值(0.3 0.7)范围内, 描 述了在MEPs时期的细胞命运决定过程. 通过进一步 将不稳定状态 $\mathrm{U} 4$ 和 $\mathrm{U} 5$ 的因子表达值与人类血液发育 样本中两类祖细胞的RNA-seq数据 ${ }^{[48}$ 进行对比, 发现 两者符合的程度分别为 $75 \%, 62.5 \%$. 这一结果证明了 内源性网络本身所产生的不稳定或过渡状态对应着 发育过程中细胞的中间状态.

(4) 网络的动力学分析揭示细胞命运决策过程及 相关预测. 为了描述细胞命运决定的动力学过程, 本 文进一步对网络进行局域扰动, 以研究不同态之间的 相互连接和可能的转化途径 (见材料与方法). 通过总 结对所有不稳态的扰动结果, 将每一个不稳定平衡点 扰动后演化到的稳态与网络计算得到的稳态进行对 比, 本文构建了内源性网络发育景观拓扑图, 以描述 不稳定状态与稳态之间的连接如图 4 , 从而讨论细胞 命运之间的联系和相互转换过程.

本文对实验中基因操作进行对应的ODE网络动力 学模拟, 发现在对网络节点表达值进行相应的处理后, 可以成功模拟出实验中观测到的表型变化. 如表3所 示, 当系统处于MEPs状态, 即不稳定平衡点U5时, 使因 子PU.1暂时性高表达, 发现在因子的相互作用下, 系统 

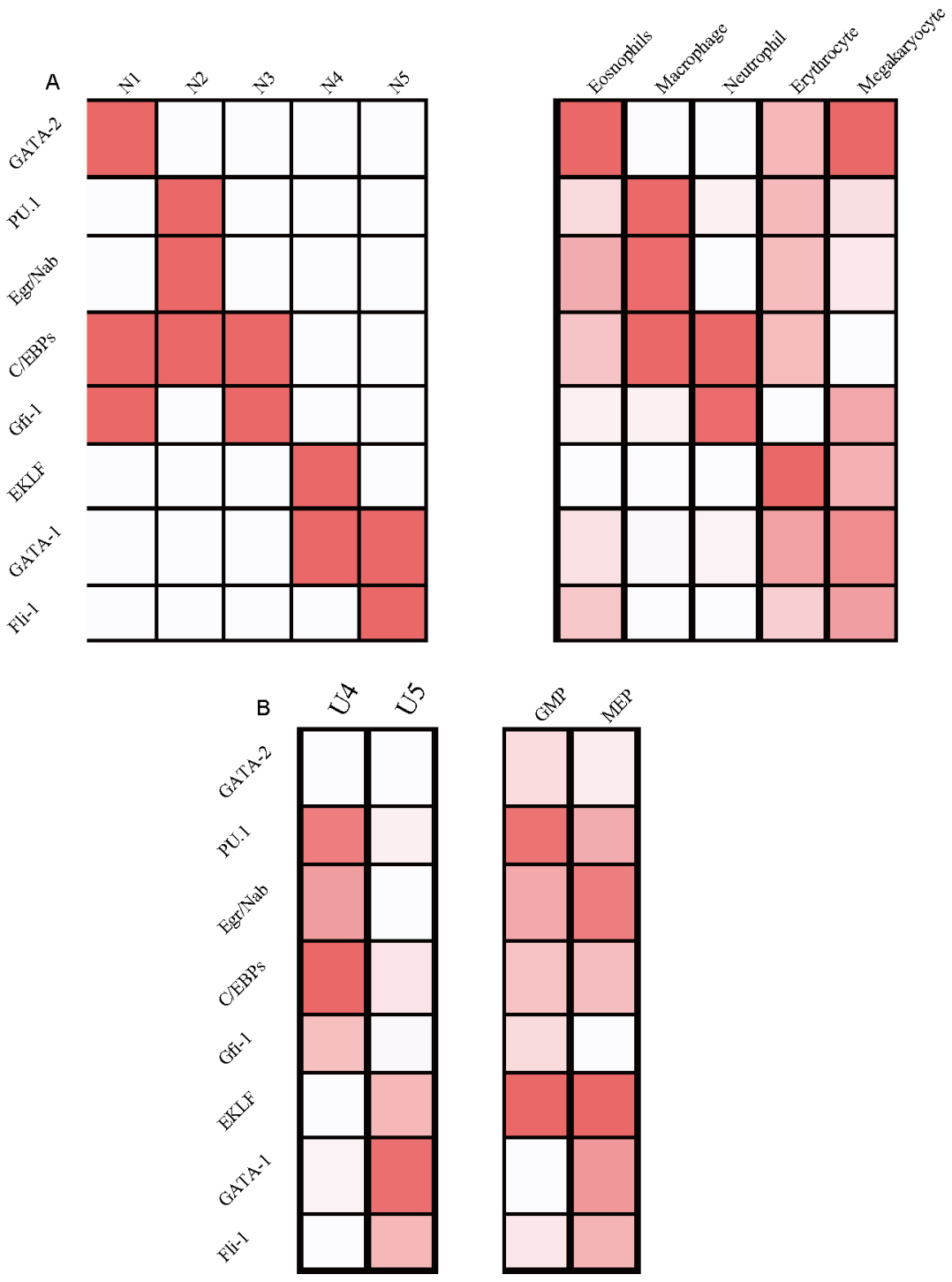

图 3 网络计算得到的稳态与取自人体血液组织不同血细胞的RNA-seq相应表达数据的对比图(网络版彩图) A: 将网络计算得到的稳态与来自人类血液组织样本中分离的不同血细胞的RNA-seq表达数据进行对比, 发现 5 个稳态与 5 种不同的血细 胞在转录因子表达层次上达到 70\%左右的对应; B: 不稳定状态U4, U5 与 GMPs及MEPs的表达达到 60\%左右的对应. 深色代表因子高 表达, 浅色代表因子低表达

表 3 髓系细胞转分化现象在网络中的模拟 ${ }^{a)}$

\begin{tabular}{ccccc}
\hline Original cell types & Manipulation & Resulting cell type & Reference & Molecular dynamics \\
\hline MEP & Inducible PU.1 * expr. & Myeloid cells & {$[49]$} & 图 5A \\
GMP & Constant GATA1 * expr. & MEP & {$[50]$} & 图 5B \\
Erythroid & Constant PU.1 ${ }^{*}$ expr. & Monocyte & {$[51]$} & 图 5C \\
Eosinophil & Constant GATA1 *expr. & MEP & {$[52]$} & 图 5D \\
\hline
\end{tabular}

a) *expr.: 表达 


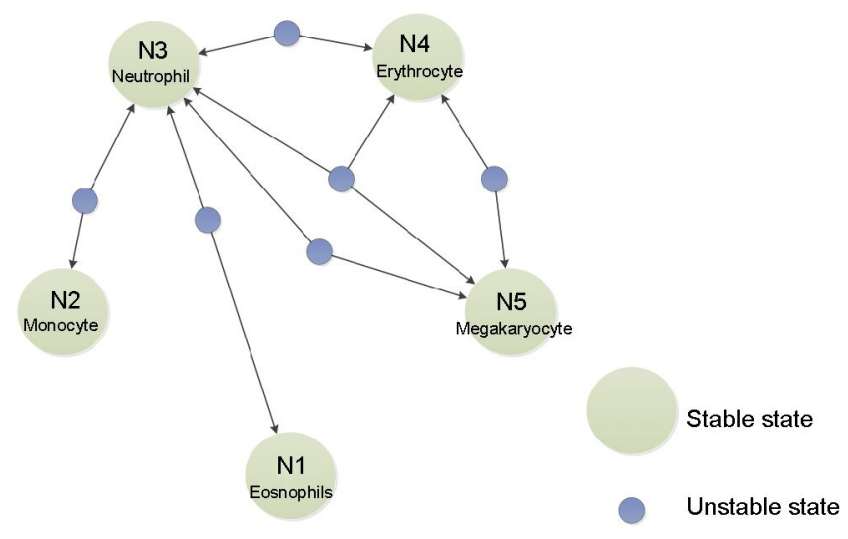

图 4 内源性网络动力学计算得到的发育景观拓扑图 (网络版彩图)

大圆表示网络中计算得到的稳态, 对应着髓系细胞发育产生的 不同细胞类型. 小圆表示不稳定或过渡状态, 对应着细胞类型转 换过程中可能经历的一些中间状态. 箭头表示了网络局域扰动 结果, 即系统在每个不稳定或过渡状态下经扰动后演化到相应 的一组稳态的过程

逐步演化至代表单核巨噬细胞的稳态 N4; 当系统处 于GMP即不稳定平衡点U4时, 使因子GATA-1 持续性 高表达, 发现系统逐步演化至代表MEP的不稳定平 衡点U5附近; 当在代表嗜酸性粒细胞的稳态N1中使 GATA-1持续性高表达, 发现系统也会逐步向MEP不稳 定平衡点靠近; 当在代表红细胞的稳态 $\mathrm{N} 2$ 中持续性高 表达PU.1时, 发现系统会演变到类似于单核-巨噬细胞 的状态. 通过与文献资料进行对比, 结果表明网络动 力学得到的态转换预测可以用于解释已有的细胞命 运转换实验观测(表3). 同时, 内源性网络动力学也进 一步预测了相应的细胞命运转化过程中, 各谱系特异 性转录因子随时间的变化过程(图5). 模型结果表明, 在细胞类型转化过程中, 因子的变化具有很强的非线 性, 一些因子在转化过程中出现先增后减的现象, 预 示了在细胞类型转化前后一些没有明显含量变化的 因子, 在细胞类型转化的过程中可能依然起到了非常 重要的作用. 内源性网络动力学模型结果为解读细胞 命运转化过程中分子调控关系和解读实验观测提供 新的启示.

\section{3 讨论}

从早期髓系细胞分化相关的内源性网络出发, 本 文讨论了包括红细胞、巨核细胞、两种粒细胞和单
核-巨噬细胞在内不同细胞命运决定过程. 经查阅文 献, 本文总结早期髓系细胞发育相关的转录调控因子 和调控关系, 构建了粗粒内源性网络. 对网络进行定 量分析和动力学模拟, 结果表明, 在合理的参数范围 和定量描述下, 一些状态始终在动力系统中稳定存在. 基于模型结果, 本文预测这些由网络结构本身所决定 的状态对应着不同的细胞类型, 并进一步预测了每种 状态下因子的表达或活性情况; 通过与独立发表的人 类血细胞RNA-seq表达数据进行对比, 发现网络动力 学预测的状态对应着不同的细胞类型: 稳态对应着 5 种具有特定功能的成熟细胞类型, 不稳定状态对应着 细胞发育的中间状态. 对不稳定状态进行局域扰动, 得到了态与态之间的连接及可能的转化路径, 并构建 了血液系统髓系细胞发育相关的景观拓扑图. 本文进 一步对态与态之间的转化进行动力学模拟, 发现从网 络动力学的角度可以解释在实验中特定遗传学操作 下观测到的分化和转分化现象, 并预测了相应过程中 关键的转录因子随时间的动力学变化, 从而为干细胞 诱导调控和细胞转分化现象提供新的理解.

在这一步的工作中, 粗粒内源性网络描述了转录 层次髓系细胞命运决定的核心调控网络, 并从动力学 角度解释了实验中观测到的态与态之间的转化过程. 然而, 在目前的网络中, 还没有办法回答更进一步的 问题, 如造血发育过程中不同细胞功能如细胞分裂, 调亡和分化之间的相互调控等. 在这个意义下, 目前 的髓系细胞发育内源性网络模型可在多个层次进行 进一步拓展. 在网络模型中加入更多的调控关系, 如 信号转导通路, 细胞因子的诱导机制以及细胞与微环 境之间的相互作用, 能够帮助进一步理解分化发育过 程中细胞根据内外环境的状况决定命运的过程, 细胞 数量分布及组织稳态平衡等问题.

总而言之, 本文从粗粒内源性网络动力学特性出 发, 提出了一个高维景观拓扑图作为定量发育模型来 讨论造血发育过程中细胞命运决定过程, 从稳态和过 渡状态的角度对细胞类型进行初步分类, 从机制上解 释了主要细胞类型的转换过程, 并预测了相应过程中 的分子动力学变化. 这一理论框架将分子调控机制与 细胞表型联系在一起, 为理解正常发育过程中组织稳 态的建立和维持及一些恶性肿瘤和再生障碍性疾病 的发生发展奠定了基础. 

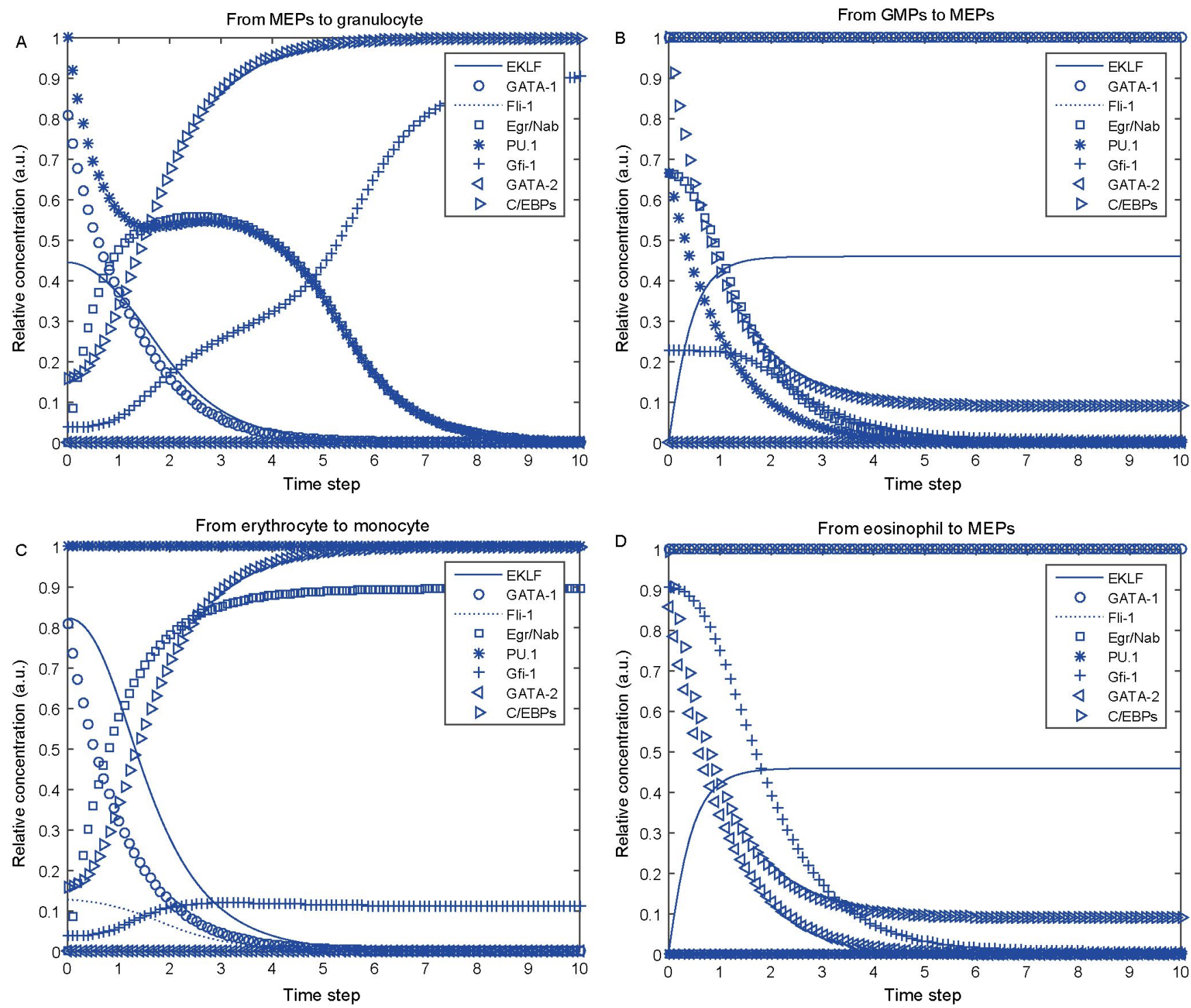

图 5 髓系细胞命运决定过程中因子的动力学变化 (网络版彩图)

A: 当系统处于MEPs状态, 即不稳定平衡点U5时, 使因子PU.1暂时性高表达, 网络因子随时间的动力学变化; B: 当系统处于 GMP即不稳定平 衡点U4时, 使因子GATA-1 持续性高表达, 网络因子随时间的动力学变化; C: 当在代表嗜酸性粒细胞的稳态N1中使GATA-1 持续性高表达, 网络因子随时间的动力学变化; D: 当在代表红细胞的稳态N2中持续性高表达PU.1时, 发现系统会演变到类似于单核-巨噬细胞的状 态. 图中横轴代表模拟的时间步数, 纵轴代表因子的相对活性

致谢感谢上海交通大学医学院附属瑞金医院血液研究所任瑞宝教授、中国科学院上海生命科学研究院生物化 学与细胞生物学研究所刘小龙教授、美国加州大学尔湾分校数学与生物医学工程系聂青教授对本工作的建设性意 见和明辨性讨论, 感谢上海交通大学敖平系统生物实验室徐岷涓和朱晓梅老师, 上海交通大学雷雪、李斯特、王俊 强、程润坦等同学在研究中的帮助. 


\section{参考文献}

1 Orkin S H, Zon L I. Hematopoiesis: an evolving paradigm for stem cell biology. Cell, 2008, 132: 631-644

2 Iwasaki H, Akashi K. Hematopoietic developmental pathways: on cellular basis. Oncogene, 2007, 26: 6687-6696

3 Cantor A B, Orkin S H. Hematopoietic development: a balancing act. Curr Opin Genet Dev, 2001, 11: 513-519

4 Waddington C H. The Strategy of the Genes. London \& New York: Routledge, 2014

5 Ao P. Global view of bionetwork dynamics: adaptive landscape. J Genet Genomics, 2009, 36: 63-73

6 Ao P, Galas D, Hood L, et al. Cancer as robust intrinsic state of endogenous molecular-cellular network shaped by evolution. Med Hypotheses, 2008, 70: 678-684

7 Wang G, Zhu X, Hood L, et al. From Phage lambda to human cancer: endogenous molecular-cellular network hypothesis. Quant Biol, 2013, 1: $32-49$

8 苏杭, 王高伟, 朱晓梅, 等. 复杂疾病的系统医学视角: 内源性网络理论. 自然杂志, 2015, 37: 448-455

9 Wang G, Zhu X, Gu J, et al. Quantitative implementation of the endogenous molecular-cellular network hypothesis in hepatocellular carcinoma. Interf Focus, 2014, 4: 20130064-20130064

10 Li S, Zhu X, Liu B, et al. Endogenous molecular network reveals two mechanisms of heterogeneity within gastric cancer. Oncotarget, 2015, 6: $13607-13627$

11 Yuan R, Zhu X, Radich J P, et al. From molecular interaction to acute promyelocytic leukemia: calculating leukemogenesis and remission from endogenous molecular-cellular network. Sci Rep, 2016, 6: 24307

12 Wang G, Su H, Yu H, et al. Endogenous network states predict gain or loss of functions for genetic mutations in hepatocellular carcinoma. J R Soc Interf, 2016, 13: 20151115

13 Yuan R, Ao P. Beyond Itô versus stratonovich. J Stat Mech, 2012, doi: 10.1088/1742-5468

14 Ao P, Galas D, Hood L, et al. Towards predictive stochastic dynamical modeling of cancer genesis and progression. Interdiscip Sci Comput Life Sci, 2010, 2: 140-144

15 Hanahan D, Weinberg R A. The hallmarks of cancer. Cell, 2000, 100: 57-70

16 Ao P. Orders of magnitude change in phenotype rate caused by mutation. Cell Oncol, 2007, 29: 67-69

17 Hanahan D, Weinberg R A. Hallmarks of cancer: the next generation. Cell, 2011, 144: 646-674

18 Rosenbauer F, Tenen D G. Transcription factors in myeloid development: balancing differentiation with transformation. Nat Rev Immunol, 2007, 7: $105-117$

19 Laiosa C V, Stadtfeld M, Graf T. Determinants of lymphoid-myeloid lineage diversification. Annu Rev Immunol, 2006, 24: 705-738

20 Graf T, Enver T. Forcing cells to change lineages. Nature, 2009, 462: 587-594

21 Tapscott S J, Davis R L, Thayer M J, et al. MyoD1: a nuclear phosphoprotein requiring a Myc homology region to convert fibroblasts to myoblasts. Science, 1988, 242: 405-411

22 Takahashi K, Yamanaka S. Induction of pluripotent stem cells from mouse embryonic and adult fibroblast cultures by defined factors. Cell, 2006, 126: $663-676$

Grass J A, Boyer M E, Pal S, et al. GATA-1-dependent transcriptional repression of GATA-2 via disruption of positive autoregulation and domainwide chromatin remodeling. Proc Natl Acad Sci USA, 2003, 100: 8811-8816

25 Walsh J C, DeKoter R P, Lee H J, et al. Cooperative and antagonistic interplay between PU.1 and GATA-2 in the specification of myeloid cell fates. Immunity, 2002, 17: 665-676

26 Weiss M J, Keller G, Orkin S H. Novel insights into erythroid development revealed through in vitro differentiation of GATA-1 embryonic stem cells. Gene Dev, 1994, 8: 1184-1197

27 Okuno Y, Huang G, Rosenbauer F, et al. Potential autoregulation of transcription factor PU.1 by an upstream regulatory element. Mol Cell Biol, 2005, 25: 2832-2845

Orkin S H. Diversification of haematopoietic stem cells to specific lineages. Nat Rev Genet, 2000, 1: 57-64

29 Laslo P, Spooner C J, Warmflash A, et al. Multilineage transcriptional priming and determination of alternate hematopoietic cell fates. Cell, 2006, 126: $755-766$

30 Spooner C J, Cheng J X, Pujadas E, et al. A recurrent network involving the transcription factors PU.1 and Gfil orchestrates innate and adaptive immune cell fates. Immunity, 2009, 31: 576-586

31 Querfurth E, Schuster M, Kulessa H, et al. Antagonism between C/EBPbeta and FOG in eosinophil lineage commitment of multipotent hematopoietic progenitors. Gene Dev, 2000, 14: 2515-2525 
Doré L C, Crispino J D. Transcription factor networks in erythroid cell and megakaryocyte development. Blood, 2011, 118: 231-239 Siatecka M, Bieker J J. The multifunctional role of EKLF/KLF1 during erythropoiesis. Blood, 2011, 118: 2044-2054

Yu C, Cantor A B, Yang H, et al. Targeted deletion of a high-affinity GATA-binding site in the GATA-1 promoter leads to selective loss of the eosinophil lineage in vivo. J Exp Med, 2002, 195: 1387-1395

Zhang P, Zhang X, Iwama A, et al. PU. 1 inhibits GATA-1 function and erythroid differentiation by blocking GATA-1 DNA binding. Blood, 2000, 96: 2641-2648

Starck J, Cohet N, Gonnet C, et al. Functional cross-antagonism between transcription factors FLI-1 and EKLF. Mol Cell Biol, 2003, 23: 1390-1402

Wang R S, Saadatpour A, Albert R. Boolean modeling in systems biology: an overview of methodology and applications. Phys Biol, 2012, 9: 055001

Bornholdt S. Boolean network models of cellular regulation: prospects and limitations. J R Soc Interf, 2008, 5: S85-S94

von Dassow G, Meir E, Munro E M, et al. The segment polarity network is a robust developmental module.. Nature, 2000, 406: 188-192

Barkai N, Leibler S. Robustness in simple biochemical networks. Nature, 1997, 387: 913-917

Ideker T, Galitski T, Hood L. A new approach to decoding life: systems biology. Annu Rev Genomics Hum Genet, 2001, 2: 343-372

Waddington C H. Canalization of development and the inheritance of acquired characters. Nature, 1942, 150: 563-565

Lei J, Levin S A, Nie Q. Mathematical model of adult stem cell regeneration with cross-talk between genetic and epigenetic regulation. Proc Natl Acad Sci USA, 2014, 111: E880-E887

4 Wang P, Song C, Zhang H, et al. Epigenetic state network approach for describing cell phenotypic transitions. Interf Focus, 2014, 4: 20130068-20130068

5 Li C, Hong T, Nie Q. Quantifying the landscape and kinetic paths for epithelial-mesenchymal transition from a core circuit. Phys Chem Chem Phys, 2016, 18: 17949-17956

Dahl R, Walsh J C, Lancki D, et al. Regulation of macrophage and neutrophil cell fates by the PU.1:C/EBP $\alpha$ ratio and granulocyte colonystimulating factor. Nat Immunol, 2003, 4: 1029-1036

Iwasaki H, Mizuno S, Arinobu Y, et al. The order of expression of transcription factors directs hierarchical specification of hematopoietic lineages. Gene Dev, 2006, 20: 3010-3021

8 Novershtern N, Subramanian A, Lawton L N, et al. Densely interconnected transcriptional circuits control cell states in human hematopoiesis. Cell, 2011, 144: 296-309

Nerlov C, Graf T. PU.1 induces myeloid lineage commitment in multipotent hematopoietic progenitors. Gene Dev, 1998, 12: 2403-2412

Kulessa H, Frampton J, Graf T. GATA-1 reprograms avian myelomonocytic cell lines into eosinophils, thromboblasts, and erythroblasts. Gene Dev, 1995, 9: 1250-1262

51 Yamada T, Kihara-Negishi F, Yamamoto H, et al. Reduction of DNA binding activity of the GATA-1 transcription factor in the apoptotic process induced by overexpression of PU.1 in murine erythroleukemia cells. Exp Cell Res, 1998, 245: 186-194

52 Nerlov C, Querfurth E, Kulessa H, et al. GATA-1 interacts with the myeloid PU. 1 transcription factor and represses PU. 1-dependent transcription. Blood, 2000, 95: 2543-2551 


\title{
Coarse-grained endogenous network predicts main dynamical sequences in myeloid differentiation and trans-differentiation
}

\author{
SU Hang ${ }^{1}$, WANG GaoWei ${ }^{1}$, YUAN RuoShi ${ }^{2} \&$ AO Ping ${ }^{1,2,3,4 *}$ \\ 1 Key Laboratory of Systems Biomedicine (Ministry of Education), Shanghai Center \\ for Systems Biomedicine, Shanghai Jiao Tong University, Shanghai 200240, China; \\ 2 School of Biomedical Engineering, Shanghai Jiao Tong University, Shanghai 200240, China; \\ 3 State Key Laboratory for Oncogenes and Related Genes, Shanghai Cancer Institute, \\ Shanghai Jiao Tong University School of Medicine, Shanghai 200240, China; \\ 4 Shanghai Center for Quantitative Life Sciences and Physics Department, Shanghai University, Shanghai 200444, China
}

\begin{abstract}
Revealing the molecular dynamics of cell fate determination is fundamental to our understanding of stem cell biology, tissue homeostasis and disease genesis. In this work, we constructed a coarse-grained endogenous network by integrating the accumulated molecular knowledge. Quantifying this coarse-grained endogenous network, we classified myeloid cell types by the structurally robust stable states generated from the endogenous network. We further predicted a series of intermediate states (transition states) in the cell-fate transition process. Accordingly, we established a quantitative scheme from the endogenous network dynamics to understand the early myelopoiesis. It not only explained the trans-differentiation events upon specific gene manipulation, but also predicted the molecular dynamics along each cell fate transition process. The quantitative scheme of early myelopoietic development can lead to a better understanding of the establishment and maintenance of tissue homeostasis and further contribute to elucidating malignant or aplastic diseases genesis systematically.
\end{abstract}

hematopoietic development, landscape, endogenous network, topology, stable states, transition states, dynamical modeling

doi: $10.1360 / \mathrm{N} 052016-00309$ 